\title{
Miller Fisher syndrome following BNT162b2 mRNA coronavirus 2019 vaccination
}

\author{
${\text { Yamato Nishiguchi }{ }^{1 *} \text { D, Hirofumi Matsuyama }}^{1}$, Kuniko Maeda ${ }^{2}$, Akihiro Shindo $^{1}$ and Hidekazu Tomimoto ${ }^{1}$
}

\begin{abstract}
Background: The coronavirus disease 2019 (COVID-19) pandemic, caused by severe acute respiratory syndrome coronavirus 2 (SARS-CoV-2), began in late 2019. One of the vaccines approved against COVID-19 is the BNT162b2 mRNA COVID-19 vaccine (Pfizer/BioNTech).

Case presentation: We present the case of a 71-year-old man with no history of the SARS-CoV-2 infection or any recent viral or bacterial illnesses who presented with bilateral oculomotor palsy and limb ataxia after BNT162b2 mRNA COVID-19 vaccination. The diagnosis of Miller Fisher syndrome (MFS) was established based on physical examination, brain magnetic resonance imaging (MRI), cerebrospinal fluid analysis (CSF), and positron emission tomography (PET). There was no evidence of other predisposing infectious or autoimmune factors, and the period from COVID-19 vaccination to the appearance of neurological symptoms was similar to that of other vaccines and preceding events, such as infection.

Conclusion: Guillain-Barré syndrome (GBS) and its variants after COVID-19 vaccination are extremely rare. Note that more research is needed to establish an association between MFS and COVID-19 vaccines. In our opinion, the benefits of COVID-19 vaccination largely outweigh its risks.
\end{abstract}

Keywords: Miller fisher syndrome, Guillain-Barré syndrome, SARS-CoV-2, COVID-19, Vaccination

\section{Background}

The coronavirus disease 2019 (COVID-19) pandemic, caused by severe acute respiratory syndrome coronavirus 2 (SARS-CoV-2), began in late 2019. Several vaccines, including BNT162b2 mRNA COVID-19 vaccine (Pfizer/ BioNTech), were developed and have been shown to be effective worldwide. Although vaccination is the best way to control the pandemic, the neurological complications have not been fully elucidated. The Guillain-Barré syndrome (GBS) is a peripheral immune-mediated neuropathy causing muscle weakness, sensory disturbances, and dysautonomia and often involves cranial neuropathies. Post-vaccination GBS has been reported recently

\footnotetext{
*Correspondence: yamato.nishi.1008@gmail.com

1 Department of Neurology, Graduate School of Medicine, Mie University,

Tsu, Mie 514-8507, Japan

Full list of author information is available at the end of the article
}

[1]. In this paper, we study the case of the Miller Fisher syndrome (MFS) recorded after receiving the first dose of BNT162b2 mRNA COVID-19 vaccine. We also reviewed all previous cases of GBS and its subtypes that occurred after COVID-19 vaccination.

\section{Case presentation}

A 71-year-old man with a medical history of diabetes mellitus and diabetic ophthalmoplegia (7years ago, and recovered completely) presented with headache and ocular pain 18 days after the first dose of BNT162b2 mRNA COVID-19 vaccine. The patient's symptoms progressed over the next 8 days to ptosis and ocular motility disorder. He took the second dose 3 weeks after the first vaccination, and his neurological symptoms continued to worsen after the second dose. On physical examination on admission (16 days after the second dose), the patient was vitally stable, afebrile, and on room air without signs 
of respiratory distress. Neurological examination showed bilateral ptosis, bilateral pupillary light reflex disappearance, oculomotor nerve palsy (Fig. 1), left-dominant limb ataxia, and mild ataxic gait.

The muscle strength of the limbs was normal, and the tendon reflex was not reduced. Complete blood cell count and coagulation profile were normal. Hemoglobin A1c was 7.1\%. CEA, CA19-9, IL-2 receptor, ACE, lysozyme, IgG4, MPO-ANCA, PR3-ANCA, antinuclear antibody, anti-SS-A antibody, anti-SS-B antibody, and anti-DNA antibody were negative. A lumbar puncture was performed, and the consequent cerebrospinal fluid (CSF) analysis showed normal white cell counts (1 cell/ microliter) and elevated protein at $67 \mathrm{mg} / \mathrm{L}$ (reference range, $15-40 \mathrm{mg} / \mathrm{L})$. The gram staining, culture, and cytology analysis of the CSF were negative. Brain magnetic resonance imaging (MRI) was normal except for minor venous dilation of the middle cranial fossa. Moreover, thin slice brain stem and the gadolinium-enhanced MRI showed no lesions with enhancing effects. Magnetic resonance angiography and venography were normal. Anti-ganglioside antibodies (i.e., GM1, GM2, GM3, GD1a, GD1b, GD3, GT1a, GT1b, GQ1b, Gal-C, and GT1a) in the serum were examined, and all tests resulted negative. Nerve conduction study of the patient's limbs showed normal findings. Positron emission tomography/ computed tomography (PET/CT) did not suggest the presence of malignant tumors or inflammatory diseases. Incomplete MFS was diagnosed because other diseases, such as meningeal carcinomatosis, hypertrophic dural inflammation, infection, collagen disease, cavernous sinus fistula, and the Tolosa-Hunt syndrome, which had similar symptoms, were ruled out. The patient received an intravenous immunoglobulin (IVIg) treatment. No complications were observed during or after the treatment. The patient's headache and eye pain improved after five doses of IVIg. Moreover, the light reflex, ptosis, ophthalmoplegia, limb ataxia, and ataxic gait gradually improved after the second course of IVIg was administered. All symptoms were improved completely after 1 month, and no recurrence was observed.

\section{Discussion}

This case report revealed two important clinical issues. First-MFS can occur following BNT162b2 mRNA COVID-19 vaccination, and second-the peak of the symptoms may be delayed in the common form of MFS, and the anti-ganglioside antibodies were all negative.

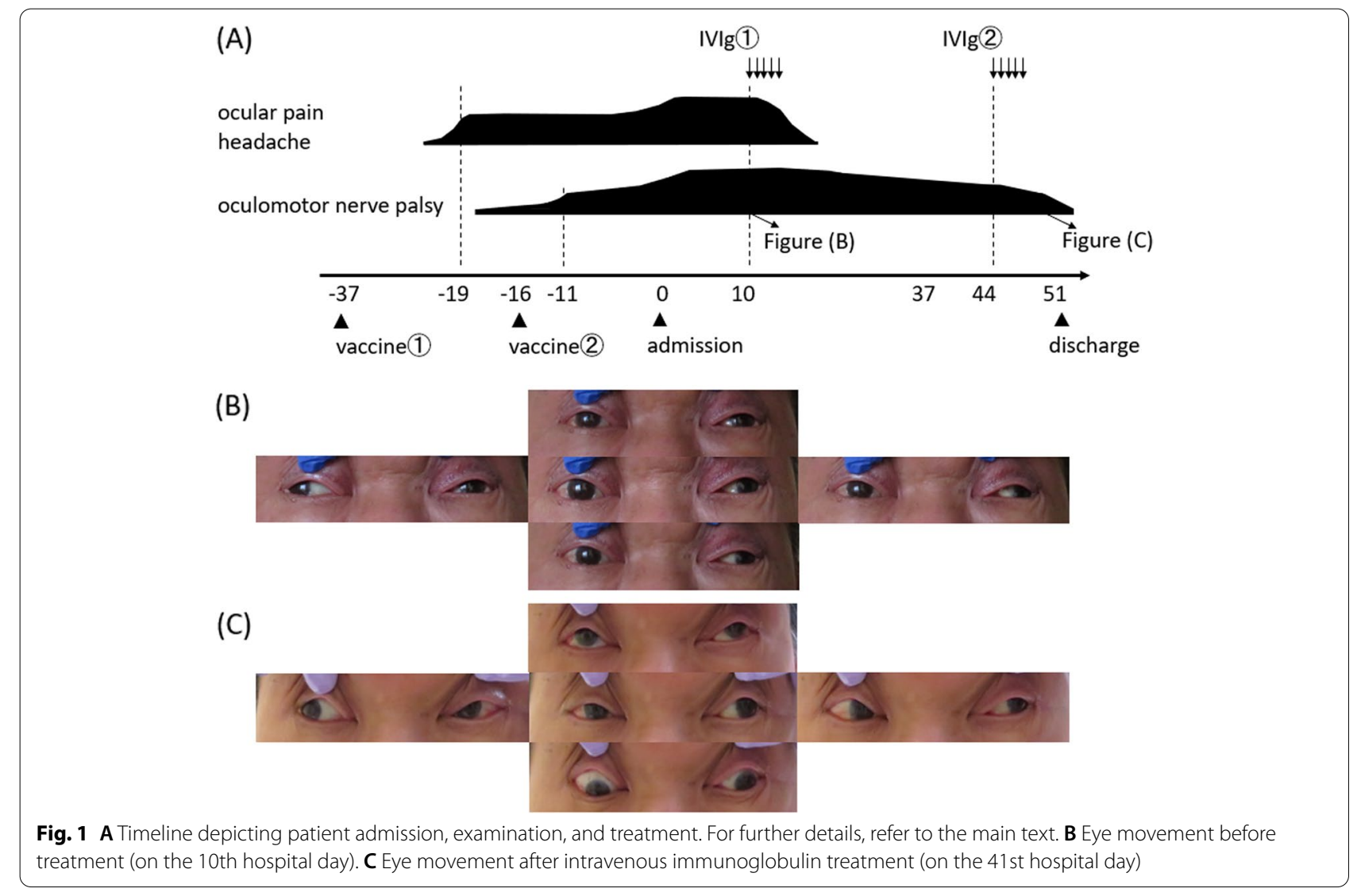


MFS is a rare variant of GBS, which is characterized by the acute onset of external ophthalmoplegia, ataxia, and loss of tendon reflexes. There exist incomplete forms of MFS, including acute ataxic neuropathy (which can be diagnosed in the absence of ophthalmoplegia) and acute ophthalmoparesis (which may occur in the absence of ataxia) [2]. MFS has been recorded to be preceded by infections similar to those preceding GBS, and in some instances, vaccination. Symptoms develop within 1-4weeks after the antecedent cause [3]. In several reports, GBS was linked with a few vaccines, namely, those against rabies, hepatitis A and B, polio, and influenza [4]. However, to our knowledge, MFS has not been reported to be associated with COVID-19 vaccination. Therefore, we investigated not only MFS but also GBS and its variant after COVID vaccination. We systematically searched in the search engine PubMed using the keywords "COVID19 " or "SARS-CoV-2", "vaccination" or "vaccine", and "Guillain-Barré syndrome", "Miller Fisher syndrome", "acute ataxic neuropathy", "acute ophthalmoparesis", "pharyngeal cervical brachial", "polyneuritis cranialis", "bilateral facial weakness with paresthesia", "acute inflammatory demyelinating polyneuropathy", "acute motor axonal neuropathy" or "acute motor sensory axonal neuropathy" to identify all studies from December 01, 2019 to July 30, 2021. Thirtyfour articles were found. Further, we selected all cases in which GBS and its variants were detected after COVID-19 vaccination. Cases that were concluded to have a negative causal relationship with COVID-19 vaccination were excluded. Consequently, 24 cases were analyzed (Table 1).

In the 25 cases studied, including the patient in this case report, the mean age was $58.4 \pm 14.7$ years (range, 20-82 years). Facial weakness was a frequent symptom observed in 19 patients (76.0\%). Four patients (16.0\%) presented with ocular motility disorder. Of the 14 cases tested for anti-ganglioside antibodies, only one test positive for it (case 7). This was significantly less than the $50-60 \%$ anti-ganglioside antibody positivity recorded in GBS [14] and 80-90\% GQ1b positivity in MFS reported in previous reports [15]. One of the cases had a history of GBS (Case 10). Finsterer has mentioned that "Neurologists should remain vigilant for a potential recurrence of GBS after vaccination with a vector-based COVID-19 vaccine" [6]. All authors concluded that "vaccines and GBS may be related, but further research is needed to establish a relationship." One of the reasons why proving the causal relationship between vaccines and GBS or MFS is difficult is that under the lockdown during the COVID-19 pandemic, the epidemic situation of many infectious diseases causing the antecedent infection of GBS or MFS is also changing. Therefore, this does not justify a negative causal relationship, if GBS or MFS did not increase from baseline after the introduction of
COVID-19 vaccines. The most common type of vaccine was Oxford-AstraZeneca. It is possible that this is due to the nature of the vaccine, which is a vector-based vaccine, but it cannot be denied that it may be biased due to the small number of reporters. Of the 25 cases, the period from vaccination to the onset of neurological symptoms was 7-22 days, similar to the duration from the preceding event to the onset of GBS in previous reports [3].

However, as previously reported by Finsterer, COVID19 infection itself can trigger GBS or MFS [6], and researchers reporting on post-vaccination GBS have emphasized that "even if the vaccine triggers GBS, it is not a reason to recommend refraining from vaccination."

Although it is unclear about the pathophysiology of GBS and MFS after COVID-19 vaccine, the report that GBS and MFS developed after COVID-19 infection are also almost negative for anti GM1 or GQ1b antibodies may helpful [16]. In other words, if both have the same pathophysiology that is not related to anti-ganglioside antibodies, it is suggested that the vaccine adjuvant and additives are not the cause of the onset but the immune response to the constituent proteins of the virus.

COVID-19 vaccination might have been responsible for the development of MFS in the patient in this case report because of the following reasons: (1) The period from vaccination to neurological symptoms coincided with the time taken for the immune response to occur, (2) neurological symptoms worsened after the second vaccination, and (3) the immunotherapy administered was effective.

Some limitations of our study should be acknowledged. First, facial nerve conduction test and Blink reflex test were not performed, because patient's consent was not obtained. Second, it is unclear whether the condition of this case is the same as that of classical MFS because anti-GQ1b antibody was negative. In addition, there was a possibility that this patient suffered from the brainstem disorder such as wall-eyed bilateral internuclear ophthalmoplegia (WEBINO) syndrome [17]. Although brainstem disorder was considered, repeated MRI scans including thin-slice brainstem evaluation of the brain were performed, showed no significant lesion.

\section{Conclusion}

We report the first case of COVID-19 vaccinationassociated MFS. However, it is difficult to deny that this result may be a coincidence in time, and therefore, no cause-and-effect relationship can be concluded at this time. However, it is indeed true that this report raises an important and timely issue. Furthermore, note that additional research is needed to establish an association between MFS and COVID-19 vaccination. We want to highlight that the risk of neurological complications or any other adverse effects associated with COVID-19 


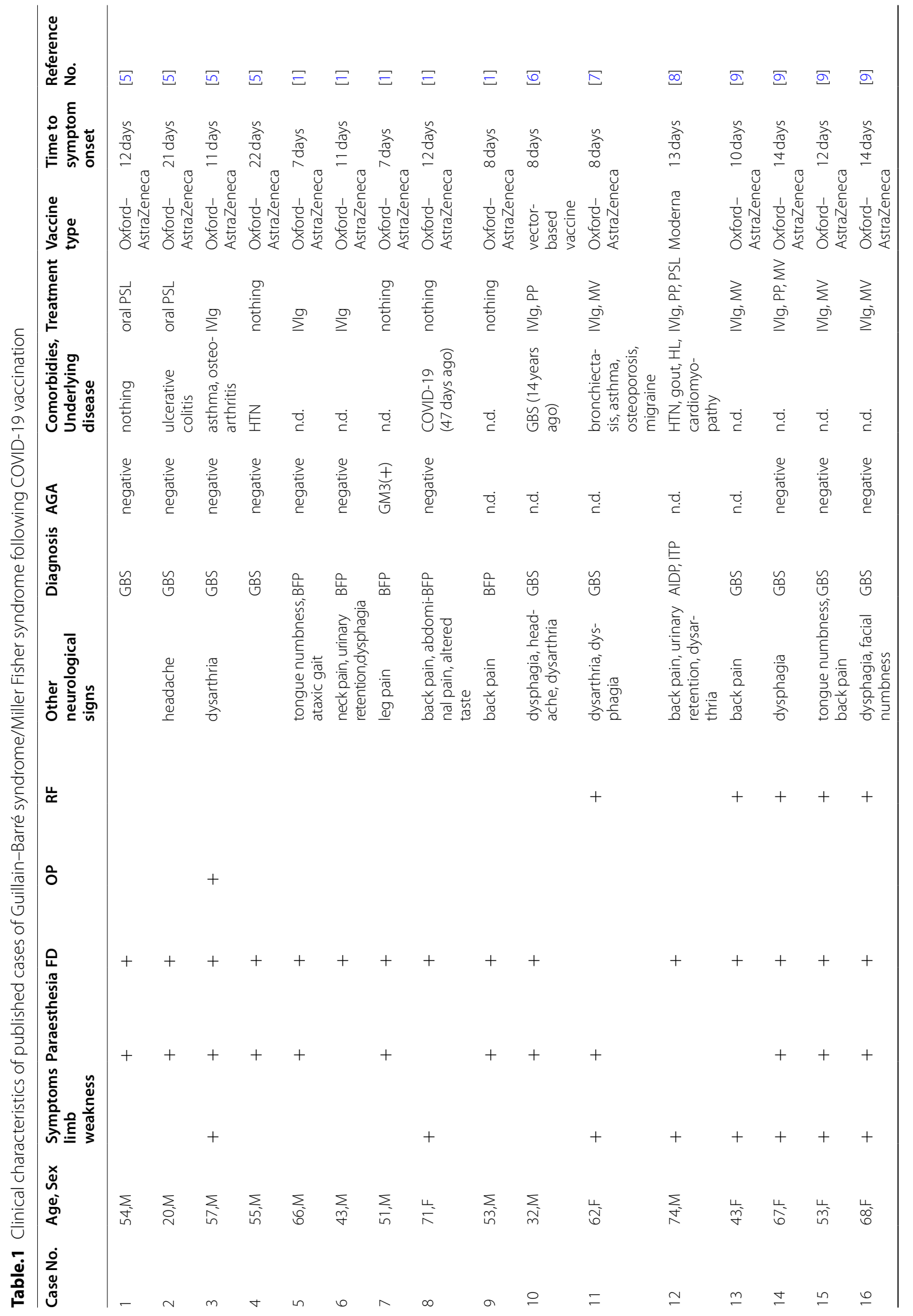




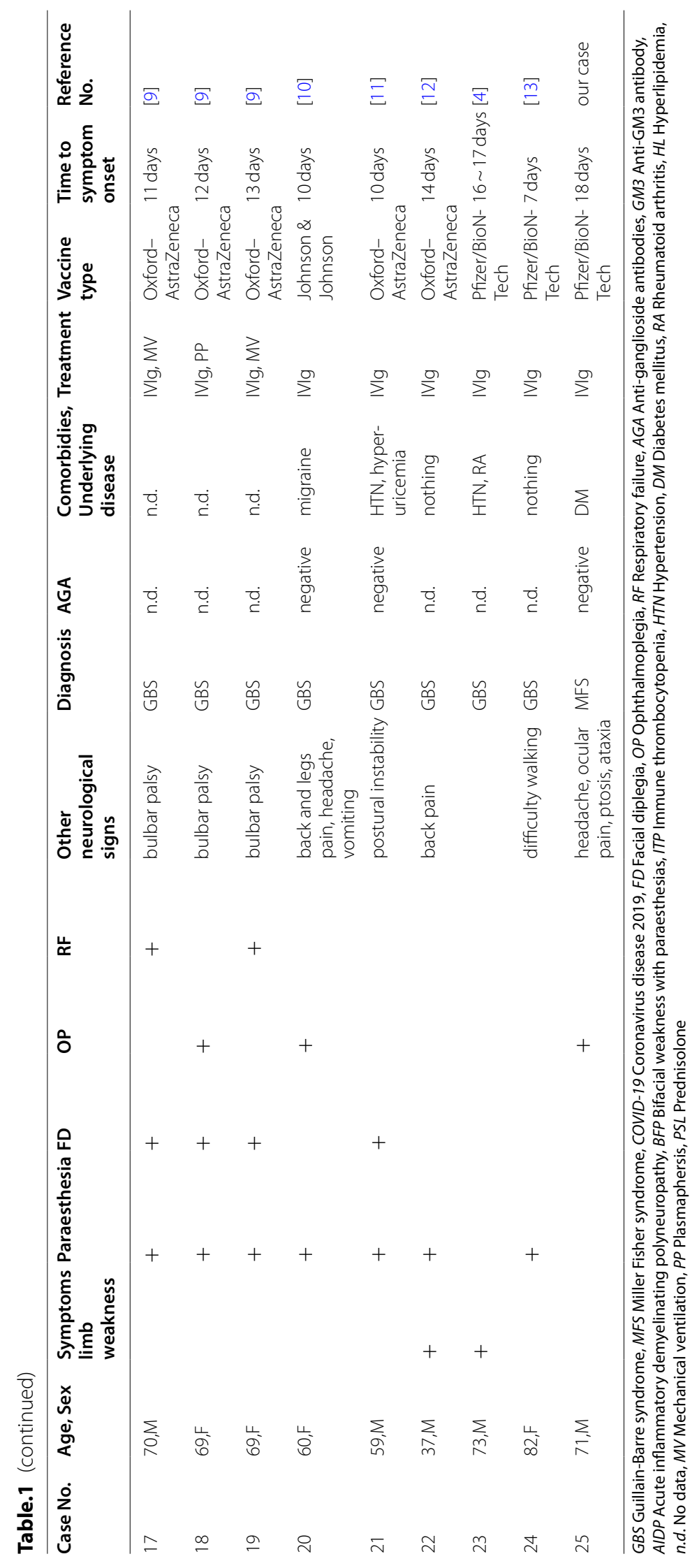


vaccination is low and the benefits of the vaccination outweigh any potential risks or side effects at both individual and social levels. However, as a clinical practice, we currently intend to highlight a precaution in patients with a history of MFS or GBS after COVID-19 vaccination for revaccination (i.e., the second dose). We support and encourage the recommendations of the Centers for Disease Control and Prevention and the guidelines by the World Health Organization for COVID-19 vaccination.

\begin{abstract}
Abbreviations
COVID-19: The coronavirus disease 2019; SARS-CoV-2: severe acute respiratory syndrome coronavirus 2; GBS: Guillain-Barré syndrome; MFS: Miller Fisher syndrome; CSF: cerebrospinal fluid; MRI: magnetic resonance imaging; PET/ $\mathrm{CT}$ : positron emission tomography/computed tomography; IVlg: intravenous immunoglobulin; WEBINO syndrome: wall-eyed bilateral internuclear ophthalmoplegia syndrome.
\end{abstract}

\section{Acknowledgements}

The authors thank Professor Susumu Kusunoki and his colleagues (Kinki University, Faculty of Medicine, Osaka, Japan) for measuring the anti-ganglioside antibodies.

The authors would like to thank Enago (www.enago.jp) for the English language review.

\section{Authors' contributions}

YN and HM performed literature review and wrote the original draft of the manuscript. KM, AS and HT supervised the writing process and revised the manuscript. All authors approved the final version for submission.

\section{Funding}

Not applicable.

\section{Availability of data and materials}

All data generated or analysed during this study are included in this published article.

\section{Declarations}

\section{Ethics approval and consent to participate}

Not applicable.

\section{Consent for publication}

Written informed consent was obtained from the patient for publication of this case report and the accompanying image.

\section{Competing interests}

The authors declare that they have no competing interests.

\section{Author details}

1 Department of Neurology, Graduate School of Medicine, Mie University, Tsu, Mie 514-8507, Japan. ${ }^{2}$ Department of Ophthalmology, Mie Prefectural Shima Hospital, Shima, Mie 517-0595, Japan.

Received: 17 September 2021 Accepted: 10 November 2021 Published online: 18 November 2021

\section{References}

1. Bonifacio GB, Patel D, Cook S, Purcaru E, Couzins M, Domjan J, et al. Bilateral facial weakness with paraesthesia variant of Guillain-Barré syndrome following Vaxzevria COVID-19 vaccine. J Neurol Neurosurg Psychiatry. 2021. https://doi.org/10.1136/jnnp-2021-327027.
2. Mori M, Kuwabara S, Yuki N. Fisher syndrome: clinical features, immunopathogenesis and management. Expert Rev Neurother. 2012;12(1):3951. https://doi.org/10.1586/ern.11.182.

3. Willison HJ, Jacobs BC, van Doorn PA. Guillain-Barré syndrome. Lancet. 2016;388(10045):717-27. https://doi.org/10.1016/s0140-6736(16)00339-1.

4. Razok A, Shams A, Almeer A, Zahid M. Post-COVID-19 vaccine GuillainBarré syndrome; first reported case from Qatar. Ann Med Surg (Lond). 2021;67:102540. https://doi.org/10.1016/j.amsu.2021.102540.

5. Allen CM, Ramsamy S, Tarr AW, Tighe PJ, Irving WL, Tanasescu R, et al. Guillain-Barré syndrome variant occurring after SARS-CoV-2 vaccination. Ann Neurol. 2021. https://doi.org/10.1002/ana.26144.

6. Finsterer J. Exacerbating Guillain-Barré syndrome eight days after vectorbased COVID-19 vaccination. Case Rep Infect Dis. 2021;2021:3619131. https://doi.org/10.1155/2021/3619131.

7. Hasan T, Khan M, Khan F, Hamza G. Case of Guillain-Barré syndrome following COVID-19 vaccine. BMJ case reports. 2021;14:6. https://doi.org/10. 1136/bcr-2021-243629.

8. Helms JM, Ansteatt KT, Roberts JC, Kamatam S, Foong KS, Labayog JS, et al. Severe, refractory immune thrombocytopenia occurring after SARSCoV-2 vaccine. J Blood Med. 2021;12:221-4. https://doi.org/10.2147/jbm. S307047.

9. Maramattom BV, Krishnan P, Paul R, Padmanabhan S, Cherukudal Vishnu Nampoothiri S, Syed AA, et al. Guillain-Barré syndrome following ChAdOx1-S/nCoV-19 vaccine. Ann Neurol. 2021. https://doi.org/10.1002/ ana.26143.

10. Márquez Loza AM, Holroyd KB, Johnson SA, Pilgrim DM, Amato AA. Guillain- Barré syndrome in the placebo and active arms of a COVID-19 vaccine clinical trial: temporal associations do not imply causality. Neurology. 2021. https://doi.org/10.1212/wnl.0000000000011881.

11. Nasuelli NA, De Marchi F, Cecchin M, De Paoli I, Onorato S, Pettinaroli R, et al. A case of acute demyelinating polyradiculoneuropathy with bilateral facial palsy after ChAdOx1 nCoV-19 vaccine. Neurol Sci. 2021. https:// doi.org/10.1007/s10072-021-05467-w.

12. Patel SU, Khurram R, Lakhani A, Quirk B. Guillain-Barre syndrome following the first dose of the chimpanzee adenovirus-vectored COVID-19 vaccine, ChAdOx1. BMJ case reports. 2021;14:4. https://doi.org/10.1136/ bcr-2021-242956.

13. Waheed S, Bayas A, Hindi F, Rizvi Z, Espinosa PS. Neurological complications of COVID-19: Guillain-Barre syndrome following Pfizer COVID-19 vaccine. Cureus. 2021;13(2):e13426. https://doi.org/10.7759/cureus.13426.

14. Kusunoki S, Kaida K, Ueda M. Antibodies against gangliosides and ganglioside complexes in Guillain-Barré syndrome: new aspects of research. Biochim Biophys Acta. 2008;1780(3):441-4. https://doi.org/10.1016/j. bbagen.2007.10.001.

15. Chiba A, Kusunoki S, Shimizu T, Kanazawa I. Serum IgG antibody to ganglioside GQ1b is a possible marker of miller fisher syndrome. Ann Neurol. 1992;31(6):677-9. https://doi.org/10.1002/ana.410310619.

16. Dufour C, Co TK, Liu A. GM1 ganglioside antibody and COVID-19 related Guillain Barre syndrome - a case report, systemic review and implication for vaccine development. Brain Behav Immun Health. 2021;12:100203. https://doi.org/10.1016/j.bbih.2021.100203.

17. Kadoya M, Onoue H, Kadoya A, Higashihara M, Ikewaki K, Kaida K. Case of wall-eyed bilateral internuclear ophthalmoplegia (WEBINO) syndrome with cerebellar ataxia and facial dysesthesia. Rinsho Shinkeigaku. 2014;54(4):317-20. https://doi.org/10.5692/clinicalneurol.54.317.

\section{Publisher's Note}

Springer Nature remains neutral with regard to jurisdictional claims in published maps and institutional affiliations. 